\title{
Entire bivariate functions of exponential type
}

\author{
Fatih Nuray · Richard F. Patterson
}

Received: 4 October 2014 / Revised: 4 February 2015 / Accepted: 6 February 2015 /

Published online: 17 February 2015

(C) The Author(s) 2015. This article is published with open access at SpringerLink.com

\begin{abstract}
In this paper we will analysis the concepts of bivariate entire complex valued functions of exponential type. To accomplish this goal, we begin with the presentation of a notion of bounded index for bivariate complex functions. Using this notion we present a series of sufficient conditions that ensure that exponential type is preserved.
\end{abstract}

Keywords Entire function $\cdot$ Bivariate function $\cdot$ Bounded index $\cdot$ Exponential type Mathematics Subject Classification $\quad 30 \mathrm{D} 10 \cdot 30 \mathrm{D} 20$

\section{Introduction}

An entire function $f(z)$ is said to be of exponential type if there is a constant $\alpha$ such that

Communicated by S. K. Jain.

F. Nuray acknowledges the support of The Scientific and Technological Research Council of Turkey in the preparation of this work.

F. Nuray

Department of Mathematics, Afyon Kocatepe University, Afyonkarahisar, Turkey

Present affiliation:

F. Nuray $(\varangle) \cdot$ R. F. Patterson

Department of Mathematics and Statistics, University of North Florida Jacksonville,

Jacksonville, FL 32224, USA

e-mail: fnuray@aku.edu.tr

R. F. Patterson

e-mail: rpatters@unf.edu 


$$
|f(z)|<\text { const. } e^{\alpha|z|}
$$

everywhere. The infimum of the set of these $\alpha$ is called the type of $f(z)$.

If $f(z, w)$ is a bivariate entire function in the bicylinder

$$
\left\{(z, w) \in \mathbb{C}^{2}:|z-a|<r_{1},|w-b|<r_{2}\right\}
$$

then at point $(a, b), f(z, w)$ have a bivariate Taylor expansion

$$
f(z, w)=\sum_{k=0}^{\infty} \sum_{l=0}^{\infty} c_{k l}(z-a)^{k}(w-b)^{l}
$$

where

$$
c_{k l}=\frac{1}{k ! l !}\left[\frac{\partial^{k+l} f(z, w)}{\partial w^{k} \partial z^{l}}\right]_{z=a ; w=b}=\frac{1}{k ! l !} f^{(k, l)}(a, b) .
$$

Similar to Gross [5] we presented in [9] the following notion of bounded index of bivariate entire function.

Definition 1 A bivariate entire function $f$ is said to be of bounded index provided that there exist integers $M$ and $N$ independent of $z$ and $w$ such that

$$
\max _{0 \leq k \leq M ; 0 \leq l \leq N}\left\{\frac{\left|f^{(k, l)}(z, w)\right|}{k ! l !}\right\} \geq \frac{\left|f^{(i, j)}(z, w)\right|}{i ! j !}
$$

for all $i=0,1,2, \ldots$ and $j=0,1,2, \ldots$ and all $z$ and $w$.

We shall say that $f$ is of index $(M, N)$ if $\mathrm{N}$ ad $\mathrm{M}$ are the smallest integers for which above inequality holds. A bivariate entire function which is not of bounded index is said to be of unbounded index. One should observe that a $\mathrm{f}$ bivariate entire function is of bounded index then there exist integers $M \geq 0, N \geq 0$ and some $C>0$,

$$
\sum_{k=0}^{M} \sum_{l=0}^{N} \frac{\left|f^{(k, l)}(z, w)\right|}{k ! l !} \geq C \frac{\left|f^{(i, j)}(z, w)\right|}{i ! j !}
$$

where $i=M+1, M+2, \ldots$ and $j=N+1, N+2, \ldots$.

In addition if the last inequality holds then

$$
\max _{0 \leq k \leq M ; 0 \leq l \leq N}\left\{\frac{\left|f^{(k, l)}(z, w)\right|}{k ! l !}\right\} \geq \frac{1}{(M+1)(N+1)} \frac{\left|f^{(i, j)}(z, w)\right|}{i ! j !}
$$

where $i, j=0,1,2,3, \ldots$ 
Slight variation of (1.1) is following

$$
\sum_{i=0}^{M} \sum_{j=0}^{N} \frac{\left|f^{(i, j)}(z, w)\right|}{i ! j !}>C \sum_{k=M+1}^{\infty} \sum_{l=N+1}^{\infty} \frac{\left|f^{(k, l)}(z, w)\right|}{k ! l !}
$$

We also consider those variation of (1.2) obtained by replacing $\left|f^{(i, j)}(z, w)\right|$ by

$$
\left(\int_{0}^{2 \pi} \int_{0}^{2 \pi}\left|f^{(i, j)}\left(r_{1} e^{\mathbf{i} \theta_{1}}, r_{2} e^{\mathbf{i} \theta_{2}}\right)\right|^{p} d \theta_{1} \theta_{2}\right)^{\frac{1}{p}}
$$

where $p$ is any positive integer. We shall show in the sequel that entire bivariate functions satisfying inequality (1.2) or any of the conditions obtained from it by the substitutions suggested above are bivariate functions of exponential type.

\section{Main result}

Let $f(z, w)$ be a bivariate function. Suppose that $0<\rho<\infty$ and let as define

$$
\tau=\limsup _{r_{1}, r_{2} \rightarrow \infty} \frac{\log M_{f}\left(r_{1}, r_{2}\right)}{r_{1}^{\rho} r_{2}^{\rho}}
$$

where

$$
M_{f}\left(r_{1}, r_{2}\right)=\sup _{|z| \leq r_{1} ;|w| \leq r_{2}}|f(z, w)|
$$

The functions which satisfy the above equality are said to be functions of exponential type $\tau$.

We can state the classical Borel [1] lemma for bivariate functions as follows:

Lemma 1 Let $T$ be a continuous nondecreasing function on $\left[r_{0}, \infty\right) \times\left[s_{0}, \infty\right)$ for some $r_{0}$ and $s_{0}$ such that $T\left(r_{0}, s_{0}\right) \geq 1$. Then

$$
T\left(r_{1}+\frac{1}{T\left(r_{1}, r_{2}\right)}, r_{2}+\frac{1}{T\left(r_{1}, r_{2}\right)}\right) \leq 4 T\left(r_{1}, r_{2}\right)
$$

for all $r_{1}$ and $r_{2}$ outside a possible exceptional set $E$ whose measure is at most 4 , that is,

$$
\iint_{(s, t) \in E \cap\left[r_{0}, \infty\right) \times\left[s_{0}, \infty\right)} d s d t \leq 4
$$

Theorem 1 Let $f(z, w)$ be a bivariate entire function and $C$ be a positive constant. If $f$ satisfies one of the following for $i=0,1,2,3, \ldots, M ; j=0,1,2,3, \ldots, N$ and for all $(z, w)$ with $|z|$ and $|w|$ sufficiently large: 
(a)

$$
\sum_{i=0}^{M} \sum_{j=0}^{N} \frac{\left|f^{(i, j)}\right|}{i ! l j !}>C \sum_{k=M+1}^{\infty} \sum_{l=N+1}^{\infty} \frac{\left|f^{(k, l)}\right|}{k ! l !}
$$

(b)

$$
\begin{aligned}
& \sum_{i=0}^{M} \sum_{j=0}^{N} \frac{\left(\int_{0}^{2 \pi} \int_{0}^{2 \pi}\left|f^{(i, j)}\left(r_{1} e^{\mathbf{i} \theta_{1}}, r_{2} e^{\mathbf{i} \theta_{2}}\right)\right|^{p} d \theta_{1} \theta_{2}\right)^{\frac{1}{p}}}{i ! j !}>C \\
& \sum_{k=M+1}^{\infty} \sum_{l=N+1}^{\infty} \frac{\left(\int_{0}^{2 \pi} \int_{0}^{2 \pi}\left|f^{(k, l)}\left(r_{1} e^{\mathbf{i} \theta_{1}}, r_{2} e^{\mathbf{i} \theta_{2}}\right)\right|^{p} d \theta_{1} \theta_{2}\right)^{\frac{1}{p}}}{k ! l !}
\end{aligned}
$$

for some integer $p$,

(c)

$$
\sum_{i=0}^{M} \sum_{j=0}^{N} \frac{\left|M_{f^{(i, j)}}\left(r_{1}, r_{2}\right)\right|}{i ! l j !}>C \sum_{i=M+1}^{\infty} \sum_{j=N+1}^{\infty} \frac{\left|M_{f^{(i, j)}}\left(r_{1}, r_{2}\right)\right|}{i ! l j !}
$$

where $M_{f^{(i, j)}}\left(r_{1}, r_{2}\right)$ is the maximum modulus of $f^{(i, j)}$ on $|z|=r_{1}$ and $|w|=r_{2}$, that is

$$
M_{f^{(i, j)}}\left(r_{1}, r_{2}\right)=\sup _{|z| \leq r_{1} ;|w| \leq r_{2}}\left|f^{(i, j)}(z, w)\right|, \quad i=0,1,2, \ldots ; \quad j=0,1,2, \ldots
$$

then $f$ is of exponential type.

Proof For convenience we choose $C=1$. The proof of other $C$ is similar. For any arbitrary bivariate entire function $F$ and any complex numbers $A$ and $B$, we have

$$
F(z, w)=\sum_{i=0}^{\infty} \sum_{j=0}^{\infty} \frac{F^{(i, j)}(A, B)}{i ! j !}(z-a)^{i}(w-b)^{j}
$$

Let $m$ and $n$ any integers, $a, b, \xi, \zeta$ complex numbers with $|\xi|=1$ and $|\zeta|=1$. Choosing $A=(m-1) \xi a, B=(n-1) \zeta b, z=m \xi+a, n \zeta+b$ and $F=f^{(i, j)}$ we obtain

$$
\left|f^{(i, j)}(a+m \xi, b+n \zeta)\right| \leq \sum_{i=0}^{\infty} \sum_{j=0}^{\infty}\left|f^{(i, j)}(a+(m-1) \xi, b+(n-1) \zeta)\right|
$$


for $m=0,1,2, \ldots ; n=0,1,2, \ldots$ Assume that (a) holds. Then (2.1) yields

$$
\left|f^{(i, j)}(a+m \xi, b+n \zeta)\right| \leq 2 \sum_{i=0}^{M} \sum_{j=0}^{N}\left|f^{(i, j)}(a+(m-1) \xi, b+(n-1) \zeta)\right|
$$

for $m=0,1,2, \ldots, M ; n=0,1,2, \ldots, N$. One observe that for $i \leq M$ and $j \leq N$

$$
\begin{aligned}
& \sum_{i=0}^{M} \sum_{j=0}^{N} \frac{\left|f^{(i+k, j+l)}(z, w)\right|}{i ! j !} \\
& \quad=\sum_{i=0}^{M} \sum_{j=0}^{N} \frac{\left|f^{(i+k, j+l)}(z, w)\right|}{i ! j !} \frac{(i+k) !(j+l) !}{(i+k) !(j+l) !} \\
& \leq \sum_{u=0}^{2 M} \sum_{v=0}^{2 N} \frac{\left|f^{(u, v)}(z, w)\right|}{u ! v !}(2 M)^{M}(2 N)^{N} \\
& \leq 2 \sum_{u=0}^{M} \sum_{v=0}^{N} \frac{\left|f^{(u, v)}(z, w)\right|}{u ! v !}(2 M)^{M}(2 N)^{N} \\
& \leq 2^{M+1} 2^{N+1} M^{M} N^{N} \sum_{i=0}^{M} \sum_{j=0}^{N} \frac{\left|f^{(i, j)}(z, w)\right|}{i ! j !} .
\end{aligned}
$$

Equations (2.2) and (2.3) yield

$$
\begin{aligned}
& \sum_{i=0}^{M} \sum_{j=0}^{N} \frac{\left|f^{(i, j)}(a+m \xi, b+n \zeta)\right|}{i ! j !} \leq(M+1)(N+1) 2^{M+1} 2^{N+1} \\
& \quad \times \sum_{i=0}^{M} \sum_{j=0}^{N} \frac{\left|f^{(i, j)}(a+m \xi, b+n \zeta)\right|}{i ! j !}
\end{aligned}
$$

\section{Letting}

$$
\lambda=(M+1) 2^{M+1} M^{M}, \mu=(N+1) 2^{N+1} N^{N}
$$

and using (2.4) recursively we have

$$
\sum_{i=0}^{M} \sum_{j=0}^{N} \frac{\left|f^{(i, j)}(a+m \xi, b+n \zeta)\right|}{i ! j !}<\lambda^{m} \mu^{n} \sum_{i=0}^{M} \sum_{j=0}^{N} \frac{\left|f^{(i, j)}(a, b)\right|}{i ! j !}
$$


For $|a|<1,|b|<1$, we get

$$
\sum_{i=0}^{M} \sum_{j=0}^{N} \frac{\left|f^{(i, j)}(a+m \xi, b+n \zeta)\right|}{i ! j !}<C \lambda^{m} \mu^{n}
$$

Letting $z=a+m \xi, w=b+n \zeta, r_{1}=|z|, r_{2}=|w|$ we get

$$
|f(z, w)|<C \lambda^{2 r_{1}} \mu^{2 r_{2}}
$$

Hence, f must be of exponential type.

If (c) is assumed instead of (a) the argument is almost identical to the one above. If (b) is assumed instead of (a), then letting $M_{p}\left(r_{1}, r_{2}\right)$ denote

$$
\left(\frac{1}{4 \pi^{2}} \int_{0}^{2 \pi} \int_{0}^{2 \pi}\left|f^{(i, j)}\left(r_{1} e^{i \theta_{1}}, r_{2} e^{i \theta_{2}}\right)\right|^{p}\right)^{\frac{1}{p}}
$$

we have as before

$$
M_{p}\left(r_{1}, r_{2}\right)<C \lambda^{2 r_{1}} \mu^{2 r_{2}}
$$

One readily sees, by means of Cauchy's formula that $R_{1}>r_{1}>0, R_{2}>r_{2}>0$

$$
M_{p}\left(r_{1}, r_{2}\right) \leq\left(\frac{R_{1}}{R_{1}-r_{1}}\right)^{\frac{1}{p}}\left(\frac{R_{2}}{R_{2}-r_{2}}\right)^{\frac{1}{p}} M_{p}\left(R_{1}, R_{2}\right)
$$

Furthermore it is easy to verify that, $M_{p}\left(R_{1}, R_{2}\right)$ is continuous and increases faster than any power of $r_{1}$ and $r_{2}$ whenever $f$ is transcendental.

Letting

$$
R_{1}=\frac{1}{\log M_{p}\left(r_{1}, r_{2}\right)}+r_{1}, \quad R_{2}=\frac{1}{\log M_{p}\left(r_{1}, r_{2}\right)}+r_{2}
$$

and by Lemma 1 we get for some $\epsilon>0$ that

$$
M_{f}\left(r_{1}, r_{2}\right)<M_{p}\left(r_{1}, r_{2}\right)^{1+\epsilon}
$$

outside a set of $r_{1}+r_{2}$ of finite measure. One can easly show that however that if there exist infinite sequences $\left(r_{1_{n}}\right)$ and $\left(r_{2_{n}}\right)$ such that

$$
M_{f}\left(r_{1_{n}}, r_{2_{n}}\right)>e^{C_{n}\left(r_{1_{n}}+r_{2 n}\right)}, \quad C_{n} \rightarrow \infty
$$


then there exist a set of $r_{1}+r_{2}$ of infinite measure with the same property. Thus

$$
M_{f}\left(r_{1}, r_{2}\right)<C \lambda^{2 r_{1}} \mu^{2 r_{2}}
$$

for sufficiently large $r_{1}$ and $r_{2}$, so proof is completed.

Open Access This article is distributed under the terms of the Creative Commons Attribution License which permits any use, distribution, and reproduction in any medium, provided the original author(s) and the source are credited.

\section{References}

1. Borel, E.: Sur les zeros des functions entieres. Acta Math. 20, 357-396 (1987)

2. Fricke, G.H., Shah, S.M.: Entire functions satisfying a linear differential equation. Indag. Math. 78(1), 39-41 (1975)

3. Gardner, R.B., Gavil, N.K.: Some inequalities for entire function of exponential type. Proc. Am. Math. Soc. 129(9), 2757-2761 (1995)

4. Gross, F.: Entire functions of exponential type. J. Res. Nat. Bur. Stand. Sect. B. 74B, 55-59 (1970)

5. Gross, F.: Entire function of bounded index. Proc. Am. Math. Soc. 18, 974-980 (1967)

6. Hamilton, H.J.: Transformations of multiple sequences. Duke Math. J. 2, 29-60 (1936)

7. Hayman, W.K.: Differential inequalities and local velency. Pac. J. Math. 44, 114-137 (1973)

8. Lepson, B.: Differential equations of infinite order, hyperdirichlet series and entire functions of bounded index. In: Lecture Notes: Summer Institute on Entire Functions. University of California, California (1966)

9. Patterson, R.F., Nuray, F.: Holomorphic bivariate functions of bounded index. Appl. Math. Comput. (submitted)

10. Rahman, Q.I., Tariq, Q.M.: On Bernstein's inequality for entire functions of exponential type. J. Math. Anal. Appl. 359, 168-180 (2009)

11. Shah, S.M.: A note on the derivatives of integral functions. Bull. Am. Math. Soc. 53, 1156-1163 (1947)

12. Shah, S.M.: Entire functions of bounded index. Proc. Am. Math. Soc. 19, 1017-1022 (1968)

13. Shah, S.M.: The maximum term of an entire series III. J. Math. Oxf. Ser. 19, 220-223 (1948)

14. Tariq, Q.Q.M.: Some inequalities for polynomials and transcendental entire functions of exponential type. Math. Commun. 18, 457-477 (2013) 